\section{Targeting Nuclear Transporters in Cancer: Diagnostic, Prognostic and Therapeutic Potential}

\author{
${ }^{1}$ Division of Medical Biochemistry, Department of Integrative Biomedical \\ Sciences, SAMRC/UCT Gynaecological Cancer Research Centre, Institute \\ of Infectious Disease and Molecular Medicine, Faculty of Health Sciences, \\ University of Cape Town, Cape Town, South Africa \\ ${ }^{2}$ Institute of Molecular Biology and Pathology, National Research Council \\ of Italy, C/O University of Roma "La Sapienza", Rome, Italy
}

\author{
Tamara Stelma ${ }^{1}$ \\ Alicia $\mathrm{Chi}^{1}$ \\ Pauline J. van der Watt ${ }^{1}$ \\ Annalisa Verrico ${ }^{2}$ \\ Patrizia Lavia ${ }^{2}$ \\ Virna D. Leaner ${ }^{1 *}$
}

\title{
Abstract
}

The Karyopherin superfamily is a major class of soluble transport receptors consisting of both import and export proteins. The trafficking of proteins involved in transcription, cell signalling and cell cycle regulation among other functions across the nuclear membrane is essential for normal cellular functioning. However, in cancer cells, the altered expression or localization of nuclear transporters as well as the disruption of endogenous nuclear transport inhibitors are some ways in which the Karyopherin proteins are dysregulated. The value of nuclear transporters in the diagnosis, prognosis and treatment of cancer is currently being elucidated with recent studies highlighting their potential as biomarkers and therapeutic targets. (C) 2016 IUBMB Life, 68(4):268-280, 2016
Keywords: Karyopherin/Importin; CRM1/Exportin; nuclear transport; cancer; diagnostic/prognostic cancer biomarkers; therapeutic target

\section{Introduction}

Protein trafficking is fundamental to cellular function and cell survival. Of the protein trafficking systems found in cells, access into the nucleus through the nuclear pore complex (NPC) is essential for the functioning of many proteins. Proteins smaller than 20-40 kDa can passively diffuse through the NPC while larger proteins require active facilitated transport to gain access in or out of the nucleus.

(C) 2016 International Union of Biochemistry and Molecular Biology

Volume 68, Number 4, April 2016, Pages 268-280

*Address correspondence to: Virna Leaner, Division of Medical Biochemistry, Faculty of Health Sciences, University of Cape Town, Observatory,

Cape Town 7925, South Africa. Tel: +27-21-406-6250.

Fax: + 27-21-406-6061

E-mail:virna.leaner@uct.ac.za

Conflict of interest: Nothing to report

Received 16 December 2015; Accepted 20 January 2016

DOI 10.1002/iub. 1484

Published online 11 March 2016 in Wiley Online Library

(wileyonlinelibrary.com)
Facilitated transport of numerous proteins is achieved via the action of the Karyopherin proteins. The Karyopherin superfamily consists of both the Karyopherin beta/Importin beta family and the Karyopherin alpha/Importin alpha family of adaptor proteins. The Karyopherin beta family forms the major class of soluble transport receptors. Its members interact with and import and/or export cargo proteins and certain RNAs across the nuclear envelope. Thus far there have been 20 genes encoding Karyopherin beta family members identified in the human genome including 10 proteins involved in nuclear import, seven proteins involved in nuclear export, two bidirectional transporters and one transporter that remains uncharacterised (Table 1) (40). The Karyopherin alpha family of adaptor proteins bind the nuclear localisation signal (NLS) of cargo proteins and link them to the Karyopherin beta transport protein, hence playing the role of an adaptor. Seven Karyopherin alpha isoforms are currently characterised, which are grouped into three subfamilies: A1, A2 and A3. The A1 subfamily comprises Karyopherin A2 and Karyopherin A7, the A2 subfamily includes Karyopherin A3 and Karyopherin A4 and, finally, the A3 subfamily consists of 


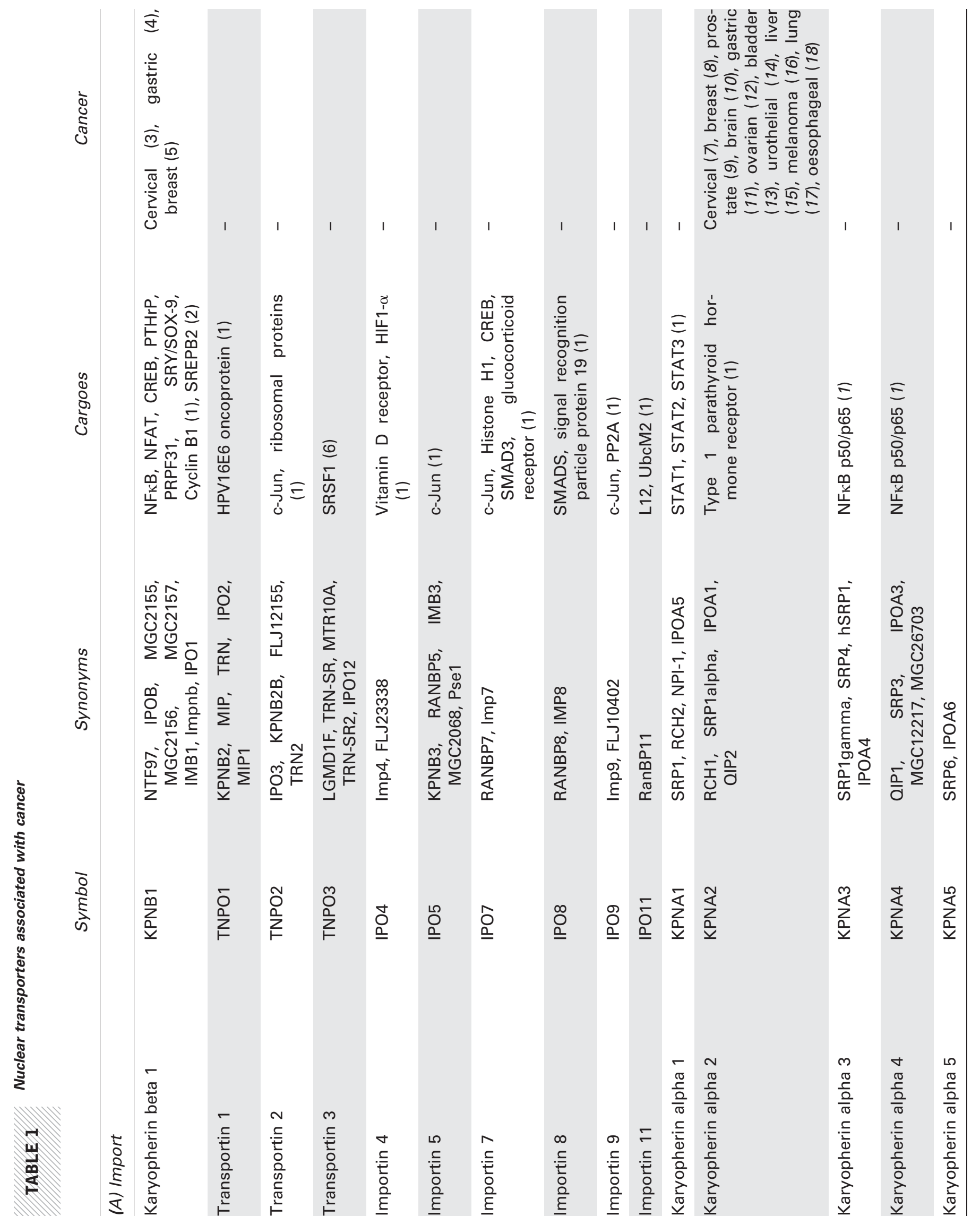




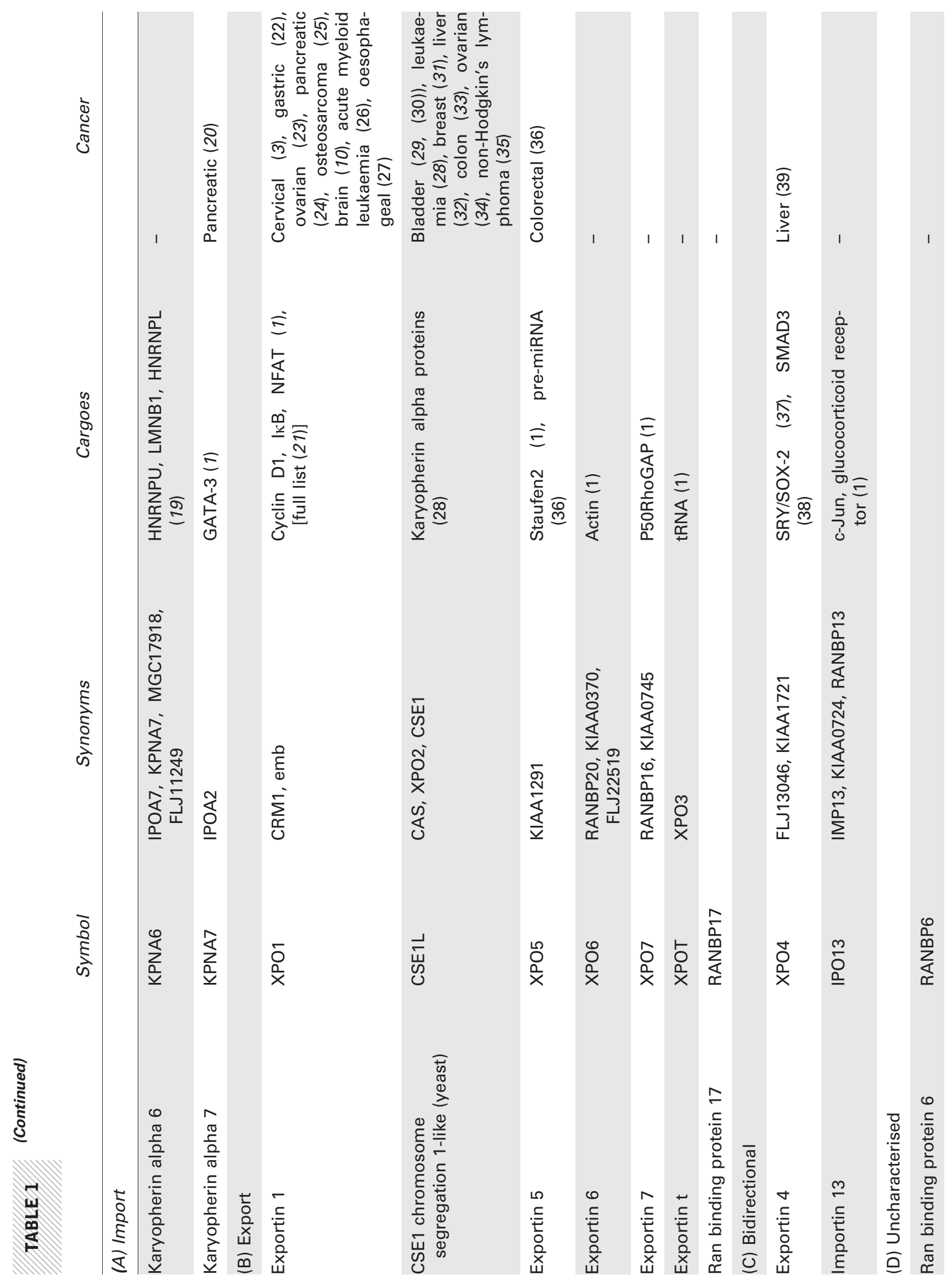




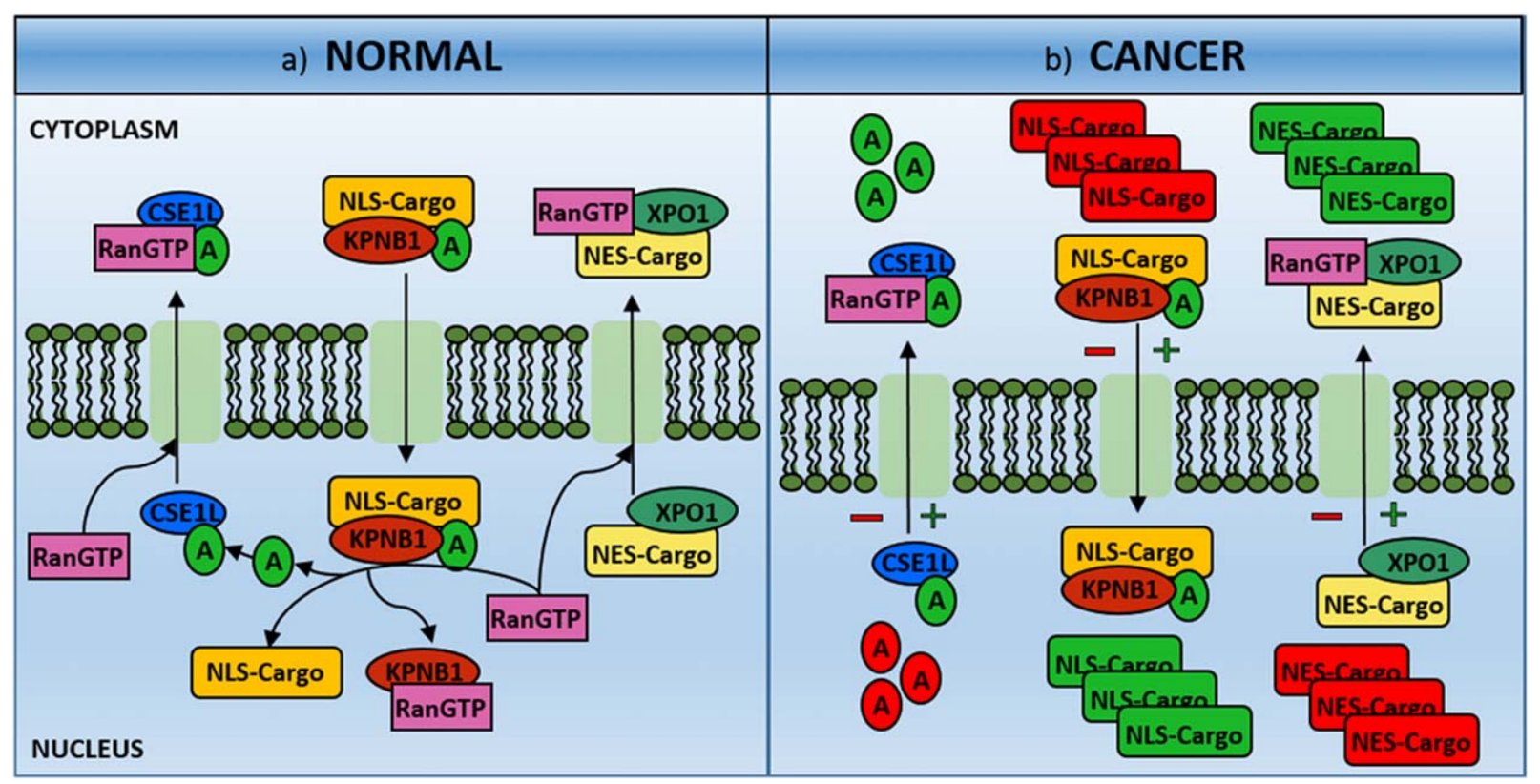

FIG 1

Proposed dysregulation of nuclear-cytoplasmic transport of cargo proteins in cancer cells in comparison to normal. (a) In normal cells protein cargo containing a nuclear localisation signal (NLS-cargo) is recognised and bound by a Karyopherin alpha (A) transport protein after which this complex is bound by Karyopherin beta 1 (KPNB1) and allowed passage through the nuclear pore complex (NPC). Upon association with RanGTP the cargo protein dissociates and the nuclear transport proteins are recycled back into the cytoplasm. Recycling of the Karyopherin alpha protein occurs through binding to CSE1L and in association with RanGTP is transported back through the NPC into the cytoplasm. Nuclear protein cargo containing a nuclear export signal (NES-Cargo) is recognised and bound by XPO1 where again in association with RanGTP is transported through the NPC into the cytoplasm. (b) In cancer cells it is proposed that the overexpression of nuclear transporters (green + ), including KPNB1, XPO1 and CSE1L leads to an increased rate of transport through the NPC. As a result their respective cargoes potentially accumulate in the cytoplasm or nucleus. Nuclear transporters can also be mislocalized (red -) rendering them nonfunctional and inhibiting transport through the NPC which causes accumulation of cargo proteins in their compartment of origin. Both overexpression of nuclear transporters and their mislocalization have been seen in cancer and associated with increased proliferation and increased survival.

Karyopherin A1, Karyopherin A5 and Karyopherin A6 (Table 1) (41).

The classical nuclear import pathway involves a Karyopherin alpha isoform recognising and binding a cargo containing a classical nuclear localisation signal (cNLS), which is then bound by Karyopherin beta 1 (KPNB1). This trimeric complex is then shuttled through the NPC. An alternative path of nuclear import involves the cargo protein bypassing the need for an adaptor and binding KPNB1 directly. Cargo proteins such as parathyroid hormone-related protein (PTHrP) and sterol regulatory-related protein (SREPB2) use this mode of nuclear import (2). Passage through the NPC is mediated by the interactions between Phe-Gly repeat-containing nucleoporins of the NPC and KPNB1. Upon arrival in the nucleus, a key event is the binding of RanGTP to the KPN/cargo complex, which promotes dissociation of the import complex, allowing the cargo to remain in the nucleus and the transport proteins to be recycled $(42,43)$. The nuclear export protein, CSE1L, is responsible for recycling the Karyopherin alphas back into the cytoplasm. XPO1, the most well-characterised export protein, binds cargoes containing a leucine-rich nuclear export signal
(NES). RanGTP stabilizes the XPO1-bound NES-cargo complex that can then pass through the NPC towards the cytoplasm. When in the cytoplasm, the hydrolysis of RanGTP to RanGDP allows the dissociation of the export protein and the cargo (Fig. 1) (44).

Although the best described role of the Karyopherin proteins is the nuclear import and export of cargo proteins, they also play an important role in mitosis and other cell cycleregulated functions including regulating the assembly of the NPC and nuclear membrane at mitotic exit as well as DNA replication in S-phase $(45,46)$. During normal cellular homeostasis, the nuclear transport system is regulated through various mechanisms including signal transduction, cell cycle, immune response, development and stress (42). Dysregulation of nuclear transport has been associated with carcinogenesis and poor prognosis in a multitude of cancers. This review aims to discuss recent work revolving around the role of key nuclear transport receptors in cancer. It will cover the dysregulation of nuclear transport pathways and focus on the potential of targeting these transporters in the diagnosis, prognosis and treatment of cancer. 


\section{Dysregulation of Nuclear Transport in Cancer}

The Karyopherin superfamily of proteins play a fundamental and indispensable role in normal cell physiology by providing the means of trafficking between the cytoplasm and the nucleus as well as in other cellular processes such as mitosis. As intracellular localization of many proteins as well as the nuclear transporters themselves has an impact on their activity status and/or function, the correct spatial arrangement of proteins at the correct time is critical. It is therefore no surprise that impaired regulation of nuclear-cytoplasmic transport associates with pathogenesis. A well-researched area is the association of nuclear transport dysregulation and cancer, which most commonly leads to the mislocalization of cancerassociated cargo proteins (Fig. 1). For example, the nuclear accumulation of the DNA repair protein, NBS1, has been shown to correlate with gastric cancer progression, while the cytoplasmic accumulation of Wilms' tumour protein, Wt1, was observed more frequently in malignant tissues than normal $(11,47)$. There are many junctions in the nuclear transport pathways at which dysregulation can occur; here we discuss mechanisms that are more frequently observed and are known to be linked to cancer. These include: (i) the altered expression of the nuclear transporters, (ii) altered localization of nuclear transporters, (iii) the disruption of endogenous nuclear transport inhibitors and (iv) the mechanistic implications of nuclear transporters in mitotic division and genetic instability.

\section{Altered Expression of the Nuclear Transporters}

The elevated expression of the Karyopherin proteins associates with the global dysregulation of protein transport and this has been observed in various types of cancer. Amongst all members of the Karyopherin family, XPO1, KPNB1, KPNA2 and CSE1L are the most frequently reported to be overexpressed in cancer (Table 1). Kuusisto et al. found that the increased expression of these Karyopherin proteins in transformed cells correlates with enhanced nuclear import and export efficiencies in transformed cells (48). The increased expression and thus transportation across the nuclear membrane is possibly a mechanism devised by cancer cells to cope with the increased metabolic and proliferative demands. The increased expression of import proteins, in particular, might allow for increased nuclear entry of proteins that have oncogenic tumour-promoting functions, for example, ERK1/2, c-Myc and E2F1 (49-51).

Whilst the link between Karyopherin overexpression and cancer has been the subject of numerous studies, few researchers have addressed the underlying mechanism leading to their overexpression. Using molecular and bioinformatics approaches, van der Watt et al. showed that the overexpression of both KPNB1 and KPNA2 is primarily due to dysregulated $\mathrm{E} 2 \mathrm{~F} / \mathrm{Rb}$ activity in cancer cells. The constitutive activation of E2F in, e.g., cervical cancer cells was found to associate with increased expression of both KPNB1 and
KPNA2 (7). It is well known that HPV infection in cervical cancer results in E2F dysregulation and thus elevated KPNB1 and KPNA2 in this cancer. The E2F/Rb pathway is disrupted in a remarkably high proportion of human cancers through other mechanisms leading to the same overexpression phenotype (52). Interestingly, Kuusisto et al. showed that the extent of overexpression of KPNB1 correlates with disease state in the MCF10 human breast tumour progression system, suggesting that its overexpression not only correlates with E2F dysregulation but can vary according to tumour progression state (5). Other mechanisms for altered Karyopherin expression have been reported. A recent study showed elevated KPNA2 expression resulting from decreased expression of microRNA-26b in epithelial ovarian carcinoma. MicroRNA-26b directly targets KPNA2 by repressing its translation and thus reduced microRNA-26b resulted in elevated KPNA2 expression (53).

Quan et al. used bioinformatic approaches to predict transcription factors that bind the promoter regions of Karyopherin beta genes and identified Sp1, NRF-2, HEN-1, RREB-1 and NFY as potential regulators of Karyopherin beta expression (54). In line with this, van der Watt et al. found that increased NFY and Sp1 expression in cervical cancer and transformed are both potential contributors to XPO1 overexpression in these cells (7). Interestingly, Kahle et al. found that NFY-A is imported into the nucleus by KPNB1 where it acts to transcribe its downstream targets (55). This finding could explain why the elevated expression of KPNB1 has been observed concomitant with elevated XPO1 in cervical and gastric cancer $(3,4,22)$. van der Watt et al. also showed that under DNA damage conditions the elevated p53 level plays a repressive role in the transcription of the xpo1 gene (3). It is not surprising then that XPO1 overexpression is commonly observed in cancer as p53 is often expressed at low levels or contains mutations in many cancers (56). Interestingly, XPO1 has also been shown to be involved in p53 nuclear export (3), suggesting that a possible feedback regulatory mechanism may exist. Under DNA damage conditions p53 expression increases and enters the nucleus, thereby repressing XPO1 expression. The reduced XP01 expression will in turn enhance p53 retention in the nucleus further repressing XP01 expression. In p53-deficient or -mutant cells the repression of XPO1 expression is relieved, likely leading to XPO1 overexpression.

The CSE1L protein, also a member of the Karyopherin family involved in recycling the KPNA adaptors into the cytoplasm, is another member commonly reported to be overexpressed in cancer including bladder, leukaemia, breast, colon, ovarian and non-Hodgkin's lymphoma $(28,31,34,35)$. Its overexpression has been attributed to gene amplification (31). More recent work by Winkler et al. demonstrated a repressive role of p53 in CSE1L and KPNA2 transcription in hepatocellular carcinoma mediated through p21 (57). As p53 loss-offunction is a frequent occurrence in cancer it is not surprising to observe overexpression of CSE1L in cancer.

The altered expression of nuclear transporters associated with cancer more often involves elevated expression; however, 
downregulation of nuclear transporters has also been linked to cancer. Exportin 4 (XPO4) is an example although the exact underlying mechanism driving its downregulation is still unknown (39).

\section{Altered Localization of Nuclear Transporters}

The ability of nuclear transporters to efficiently execute their functions depends on their ability to interact correctly with other members of the transport cycle. A truncated KPNA lacking part of the cargo-NLS-binding domain has also shown failure to interact with and transport p53 into the nucleus in breast cancer cells. Despite the KPNB1-binding domain remaining intact in the mutant KPNA, it was localized predominantly in the cytoplasm and perinuclear region as opposed to the even distribution observed in wild-type KPNA (58). This suggests that cargo recognition and binding by KPNA is a prerequisite for nuclear entry, and that pairing with KPNB1 alone is not sufficient. A similar observation was made by Melo et al., where an inactivating mutation in Exportin 5 (XP05) found in a subset of human tumours rendered it unable to export itself as well as its precursor microRNA cargoes out of the nucleus (36). As a result, the pre-microRNA processing efficiency was decreased leading to defects in microRNA production. Ultimately, failure to generate mature microRNA leads to the impairment of the post-translational regulation of target genes and altered expression profile.

Post-translational modification can also regulate protein localization, which is well demonstrated by the CSE1L protein. CSE1L is involved in more than one function inside the cell, but its role in nuclear transport is specifically carried out when localized to the nucleus (59). Its localization is phosphorylationdependent, while AKT-mediated phosphorylation is required for its nuclear localization in ovarian cancer; MEK-1-mediated phosphorylation retains it in the cytoplasm $(60,61)$. Furthermore, siRNA-mediated inhibition of CSE1L expression in ovarian cancer cells which exhibited predominant nuclear localization of CSE1L showed increased sensitivity to cisplatin treatment compared to breast and colon cancers which showed predominant cytoplasmic localization (60). This suggests that the nuclear CSE1L plays an oncogenic role and could contribute to the resistance against cytotoxic agents. The aberrant function of the upstream kinases can thus result in the incorrect localization of CSE1L and resultant pathogenesis.

\section{Disruption of Endogenous Nuclear \\ Transport Inhibitors}

Cells also use endogenous nuclear transport inhibitors to regulate nuclear trafficking. The two most well-known endogenous inhibitors are Complement Component 3 (CC3/TIP30) and Aplasia Ras Homolog Member 1 (ARH1/NOEY2). Both proteins were found to have reduced expression or absence in a wide range of cancers. Knockdown studies concluded that these proteins function to suppress the cancer phenotype, while restoration of their expression was unfavourable to cancer development. Their ability to negatively regulate nuclear transport was only recognised about a decade later $(62,63)$. Both pro-

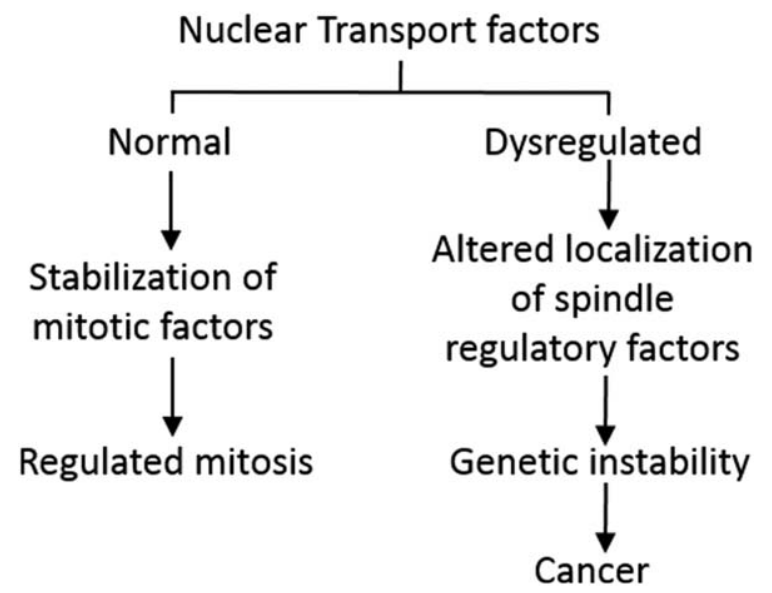

FIG 2

\begin{abstract}
Role and implications of nuclear transporters in mitosis. Under normal conditions upon cessation of nuclear transport, nuclear transport factors take on new roles in mitosis. They contribute to the stabilization of mitotic factors and regulated mitotic division. Dysregulation of the expression of nuclear transport factors has been shown to contribute to the altered localization of key spindle regulatory factors that associate with the onset of genetic instability, which is considered one of the hallmarks of cancer.
\end{abstract}

teins have been reported to interfere with nuclear import by physically interacting with members of the Karyopherin family. Binding of CC3 to the Karyopherins was RanGTPindependent and also associated with nucleoporins in vivo. Alternatively, ARH1 is thought to interact with Karyopherins through the NLS domain, thereby preventing NLS-cargo binding, and also competed with the binding of Karyopherin to Ran (64). Both inhibitors have the ability to prevent nuclear translocation of NLS-cargoes while CC3 was also able to prevent transport of M9 signal-bearing cargoes (62). The alleviation on nuclear transport and the subsequent altered cellular localization of cargo proteins has several advantages in tumour development and survival.

\section{Mechanistic Implications of Nuclear Transporters in Mitotic Division and Genetic Instability}

When nucleo-cytoplasmic transport ceases, virtually all transport factors take on new roles in mitosis, acting in the functional organisation of the mitotic spindle and in the reconstitution of the interphase nucleus at mitotic exit. Modelling studies have indicated that subtle dysregulation of the expression of transport factors readily affects mitotic division and can cause significant abnormalities in chromosome segregation, whereas nucleo-cytoplasmic transport is a more robust process $(65,66)$. Dysregulation of nuclear transport factors can therefore cause the onset of genetic instability, a cancer hallmark (Fig. 2).

The role of KPNB1 as a regulator of spindle formation and function is well described. KPNB1 generally acts by preventing the premature localisation or activity of spindle regulatory factors (67). Factors, with spindle assembly functions, regulated by KPNB1 include: the spindle pole-organizing protein NuMA 
(68), the microtubule-regulatory protein HURP (69), Rae1/ Nup98 (70), a regulator of the Aurora-A kinase maskin (71) and APC (72), among others. KPNB1's physical association and regulation of the Adenomatous polyposis coli (Apc) oncogene, itself a microtubule-promoting and mitotic spindle-associated factor, may be of particular relevance to the onset of colon cancer (72). A novel function through which KPNB1 can globally regulate the mitotic apparatus has recently emerged and concerns its ability to regulate, in concert with the APC/C ubiquitin ligase, the stability of mitotic spindle regulatory factors (73).

Transportin 1 (TNP01) has documented effects on the mitotic apparatus and on post-mitotic reorganization of the interphase nucleus, a critical process that is evidently crucial for resuming transcription and replication via the cell cycle $(74,75)$. Disruption of this process by altered TNPO1 function will also affect the genetic identity of the newly formed daughter cells after mitosis.

The CSE1L protein has predominant roles in the regulation of apoptosis, as recalled above, most probably mediated by the mislocalization of Karyopherin alpha members. It also has the ability to associate with the mitotic apparatus; altered activity of CSE1L can confer resistance to taxol but not to other chemotherapeutic drugs (76). This effect is mediated through Mek proteins, and it is particularly interesting in the light of designing new potential therapeutics $(76,77)$.

Karyopherin alpha family members, although difficult to discuss collectively, share a common functional feature that their downregulation induces substantial levels of apoptosis (78). They contribute to mitotic control by interacting with key spindle regulatory factors such as TPX2 (79). In addition, karyopherin alpha 3 has a global role in mitosis. It interacts specifically with RCC1, the guanine exchange factor for Ran, and this interaction is inhibited by mitotic phosphorylation $(78,80,81)$. This inhibition results in an increased concentration of free RCC1 and hence increased RanGTP production in mitosis.

The nuclear export protein, XPO1, has well-defined mitotic effects exerted at various levels of mitosis. These include the mitotic spindle (82); kinetochores, stabilizing microtubule/ kinetochore interactions essential for chromosome segregation (83) and centrosomes, preventing chromosome reduplication that constitutes a major threat to genome stability $(84,85)$. These effects are mediated through several mitotic targets that contain NES sequences. The interplay between XPO1 and mitotic cyclin B is interesting to note. XPO1 contributes to cyclin B localization and in turn cyclin B phosphorylates XPO1 to finely regulate its mitotic functions (86). It has also been reported that XP01's interaction with survivin is important to target the chromosomal passenger protein to kinetochores (87).

Nuclear transport plays a critical role in the functioning of many cellular processes. Because of this, cells have developed elegant systems to tightly control the nuclear transport processes. Unfortunately, within each junction in the regulatory pathway lies an opportunity for errors to occur, and indeed many of these alterations have been associated with cancer.

\section{Role of Nuclear Transporters in the Diagnosis of Cancer}

Late diagnosis of cancer is a major contributing factor to poor patient outcome. Often, by the time patients present at clinics, the cancer has already progressed to later stages and the chances of the primary tumour having already metastasised are relatively high. This highlights a need for effective tools for the early detection of cancer. The ideal biomarker would be unique to cancer and present in easily obtainable patient samples such as serum or urine. Nuclear transporters have already been shown to be upregulated at the protein level in many cancer tissues but few studies recognise these proteins as potential diagnostic biomarkers. Wang et al. investigated potential biomarker targets that were both significantly upregulated in lung cancer tissues and secreted/released from lung cancer cells. They achieved this by integrating two lung adenocarcinoma cell line secretome datasets with one adenocarcinoma microarray dataset. Using this strategy they identified KPNA2 as a potential diagnostic biomarker for adenocarcinoma. Elevated KPNA2 serum levels were confirmed in a cohort of non-small-cell lung carcinoma patients $(n=126)$ in comparison to healthy individuals $(n=64)$ (17). Similar to what was observed in lung cancer patients, KPNA2 levels in serum were also significantly upregulated in oesophageal squamous cell carcinoma patients $(n=86)$ versus healthy controls $(n=60)$ validating the potential of KPNA2 as a diagnostic biomarker (88). KPNA2 has also been identified as a potential diagnostic biomarker that can differentiate between grades of astrocytoma. This, however, still requires immunohistochemical validation. The conventional criteria for differentiating between WHO grade II and III astrocytomas is particularly difficult to define. Gousias et al. found that patients diagnosed with WHO grade II astrocytoma showed little/no nuclear KPNA2 immunostaining, whereas half of the patients diagnosed with anaplastic astrocytoma (WHO grade III) showed $\geq 5 \%$ KPNA2 staining in the nucleus (89).

The nuclear transporter, CSE1L, has also shown diagnostic potential in bladder cancer and metastatic colorectal cancer $(29,33)$. It is upregulated in bladder cancer and has been found to be secreted into the urine of these patients while it is not detected in the urine of healthy individuals (29). CSE1L has also been found to be secreted into the sera of patients with metastatic colorectal cancer (33). Thus far, no data have been published regarding the serum levels of other nuclear transport proteins in cancer. As previously mentioned, other members of the Karyopherin superfamily such as KPNB1 and XPO1 have also been shown to be upregulated in various cancers. It is thus possible that these proteins might also be secreted from cancer cells and have potential as diagnostic markers. 


\section{Nuclear Transporters as Prognostic Markers}

A study by van der Watt et al. found that KPNB1, KPNA2 and XPO1 are all upregulated in cervical cancer patient samples in comparison to normal tissue. KPNB1 and XPO1 but not KPNA2 were found to be essential for the survival of cervical cancer cells. The nuclear importer, KPNB1, and exporter, XP01, both show promise as a cancer biomarker but have not as yet been correlated to patient prognosis in cervical cancer (3). More recently, Zhu et al. found that KPNB1 was also upregulated in gastric cancer patient tissue and cells in comparison to their normal counterparts. Interestingly, Zhou et al. found XPO1 protein levels to be significantly upregulated in gastric cancer patient tissues as well, indicating that both import and export machinery is upregulated in gastric cancer. The upregulation of KPNB1 correlated positively with Ki-67 immunostaining, infiltration depth and tumour grade but not TNM stage and lymph node metastasis. On the other hand, overexpression of XPO1 positively correlated with TNM stage as well as metastasis. Both increased KPNB1 and XPO1 expression have been identified as independent prognostic factors to predict gastric cancer patient survival $(4,22)$.

XP01 has also been identified as a suitable independent prognostic marker in ovarian cancer, pancreatic cancer, osteosarcoma, brain cancer and acute myeloid leukaemia (10, 23$26,90)$. Studies on pancreatic cancer as well as osteosarcoma revealed XPO1 overexpression to be associated with increased tumour size as well as histological grade in osteosarcoma but not pancreatic cancer. For both pancreatic cancer and osteosarcoma there is evidence suggesting that increased XPO1 expression is an indicator of reduced overall and progressionfree survival $(24,25)$. In contrast, decreased expression of the nuclear export protein, XPO4, was associated with poor overall survival in patients with liver cancer suggesting XPO4 has tumour suppressor properties $(39,91)$. Although expression levels of nuclear transporters often enhances their prognostic value, in some cases so does their localisation within the cell. While the localisation of XPO1 between the nucleus and the cytoplasm showed no significant differences among gastric cancer samples, the cellular localisation of XPO1 in ovarian cancer has been correlated with different aspects of cancer progression (22). Enhanced cytoplasmic XPO1 has been associated with advanced ovarian tumour stage, poor differentiation and higher mitotic rate. Nuclear XPO1 levels have been associated with enhanced Cox-2 expression leading to poor overall patient survival (23). Nuclear XPO1 has also been correlated with pathological stage in gliomas (90). XPO1 localisation is also altered during oesophageal tumourigenesis, where it shifts from predominantly nuclear in normal tissue to nuclear and cytoplasmic in cancer tissue. This appears to occur in the early stages of disease progression (27).

The nuclear export protein, CSE1L, has been reported to have prognostic potential in bladder, liver and ovarian cancers (30, 32, (34)). Chang et al. found urothelial carcinoma patient samples to have differing cytoplasm and nuclear CSE1L staining. While cytoplasmic CSE1L staining showed no correlation to clinical manifestations, nuclear CSE1L closely correlated with poor overall survival (30). In liver cancer, CSE1L was found to be upregulated particularly in the cytoplasm of cells in comparison to normal tissue, but the association to patient outcome was not further investigated (32).

The value of nuclear transporters such as KPNB1, XPO1 and CSE1L as prognostic tools is still under investigation and data supporting this are only available for a select few cancers. In contrast, KPNA2 has been well established as a prognostic marker in various cancers including: breast cancer, brain cancer, gastric cancer, prostate cancer, ovarian cancer, bladder cancer, liver cancer, melanoma, lung cancer and oesophageal cancer $(8-11,14,15,92,93)$. Dahl et al.'s research in breast cancer was the first to identify KPNA2 as a prognostic marker (94). Since then high nuclear KPNA2 expression has been linked to poor patient outcome. Numerous studies have shown that KPNA2 expression correlates with overall and progression-free survival in patients and established KPNA2 as an independent prognostic marker when compared to other clinical data $(10,(11,13-15,89,93,95,96))$. Interestingly, KPNA2 expression also predicted the chances of metastasis as well as histological grade and clinical stage of tumours. High KPNA2 expression in non-invasive bladder cancer increased the risk of progression to a more invasive form (13).

Some malignancies have a high recurrence rate following therapeutic or surgical intervention and it is important to identify risk factors in these patients that might predict recurrence. KPNA2 expression has been identified as a marker of both early and more frequent recurrence in liver cancer while it is also a marker of PSA recurrence in prostate cancer $(9,97,98)$. In meningioma, elevated KPNA2 and XPO1 expression was observed in recurrent tumours in comparison to primary tumours and this correlated with increased recurrence rates (10).

The evidence presented here highlights that KPNA2 serves as a valuable prognostic marker throughout the progression of cancer from early-stage recurrence to chances of metastasis, as well as overall survival. While there is evidence that KPNB1, XP01, CSE1L and XP04 have shown potential as prognostic markers, this requires further investigation and validation. Evidence from the study by Gousias et al., which shows KPNA2 expression levels correlating with XPO1 expression levels in meningioma, suggests that other nuclear transporters may too have prognostic value in cancer (10).

\section{Targeting Nuclear Transporters as Cancer Therapeutics}

The upregulation of nuclear transporters and their association with poor prognosis in cancer highlights their potential as therapeutic targets. Challenges may arise though when 
TABLE 2

Nuclear transport inhibitors ${ }^{\text {a }}$

\begin{tabular}{|c|c|c|c|}
\hline Nuclear transporters & Inhibitor & Nature of the compound & Experimental status \\
\hline KPNB1 & cSN50.1 (103) & Peptide & Cell culture \\
\hline \multirow[t]{6}{*}{ KPNA/B1 } & Ivermectin (104) & Antibiotic & \\
\hline & cSN50.1 (103) & Peptide & Cell culture \\
\hline & Bimax1 (105) & Peptide & Cell culture \\
\hline & Bimax2 (105) & Peptide & Cell culture \\
\hline & Karystatin 1A (106) & Small molecule & Cell culture \\
\hline & Importazole (107) & Small molecule & Cell culture \\
\hline TNPO1 & M9M (108) & Peptide & Xenopus model \\
\hline \multirow[t]{8}{*}{ XPO1 } & Leptomycin B (109) & Antibiotic & Phase I clinical trials (discontinued) \\
\hline & Ratjadone analogs (21) & Antibiotic & Cell culture \\
\hline & Anguinomycin (21) & Antibiotic & Cell culture \\
\hline & Goniothalamin (21) & Organic molecule & Cell culture \\
\hline & Kos-2464 (21) & Small molecule & Xenograft mouse model \\
\hline & $\mathrm{N}$-azolylacrylate analogs (21) & Small molecule & HIV model \\
\hline & CBS9106 (21) & Small molecule & Xenograft mouse model \\
\hline & SINE series (110) & Small molecule & Phase I-II clinical trials \\
\hline
\end{tabular}

${ }^{a}$ As published until December 2015.

targeting cellular machinery that is active in both normal and cancer cells. However, studies have shown that cancer cells are more sensitive to nuclear transport inhibition than noncancer cells which remain viable $(3,(5,99))$. Specific knockdown using siRNA for nuclear import proteins, KPNB1 and KPNA2, and export proteins, XPO1 and CSE1L, in cancer cells frequently results in reduced proliferation and increased apoptosis $(50,57,78,96,98,100-102)$. This suggests that cancer cells may have an enhanced dependence on nuclear transporters for their increased proliferative and metabolic demands (referred to as tumour cell "addiction").

\section{Targeting Nuclear Export via XPO1}

Among the Karyopherin family members, the nuclear exporter, XP01, has so far been the most successful chemotherapeutic target already being tested in clinical trials (Table 2). Several natural products were first described as inhibitors of XP01 with Leptomycin B being the most potent. Unfortunately, Leptomycin B showed severe cytotoxic effects in phase I clinical trials and was no longer pursued for use in patients. Following that several Leptomycin B derivatives such as Anguinomycin and Kos-2464 have been synthesized in an attempt to reduce the cytotoxity while retaining the ability to inhibit nuclear export via XP01, but have as yet not entered clinical trials. A recent review by Ishizawa et al. outlines the history of XPO1 as a chemotherapeutic target in more detail (21). In 2012 the SINE (selective inhibitors of nuclear export) series of drugs were discovered through an in silico molecular modelling strategy and synthesized by Karyopharm Therapeutics (Karyopharm Therapeutics, Boston, MA) (110). These compounds are water-soluble and irreversibly modify a cysteine (Cys528) in the NES-binding groove of XPO1, thereby inhibiting the function of the protein. KPT-185 is the most potent of the series and is most commonly studied in vitro; however, KPT330/Selinexor, while nearly as potent, has more acceptable pharmacokinetics and has shown promise in phase I and II clinical trials in both haematological and solid tumours (111).

More recently, combination treatment strategies using XP01 inhibitors and currently available chemotherapeutic agents have proven to be effective in enhancing the treatment of cancer. Nuclear export inhibitors alone, while effective in causing cell death in vitro, in animal models these inhibitors while inhibiting tumour growth were less often likely to eliminate existing tumours. The combination therapy is thought to use the XP01 inhibitor to sensitise cancer cells to chemotherapeutic agents. A review by Turner et al. extensively discusses 
a range of studies providing evidence for the efficacy of XPO1 inhbitors in combination with alkylating agents, anthracyclines, BRAF inhibitors, platinum drugs, protease inhibitors and tyrosine-kinase inhibitors against both haematological and solid tumours (112). SINE compounds, KPT-185, KPT-249 and KPT-330, were able to sensitize myeloma cells to doxorubicin, bortezomib and carfilzomib and phase I/II clinical are currently underway $(112,113)$. A combination therapy using KPT330 and gemcitabine has also been found to synergistically enhance cell death in vitro and in vivo in pancreatic cancer (114).

\section{Targeting Nuclear Import via KPNB1 and KPNA}

The field of nuclear import inhibitors, while not as advanced as that of nuclear export inhibitors at present, is growing (Table 2). Most commonly, KPNB1 and the KPNA isoforms work together to transport cargoes into the nucleus, although KPNB1 can also transport cargo independently. Therefore, targeting KPNB1 may have a broader spectrum of import inhibition while targeting a single KPNA isoform would considerably increase specificity. Pioneering work in the search for Karyopherin alpha/beta 1 inhibitors was started by Lin et al. in 1995. They, among others, found that a cell-permeable peptide containing the NLS of the $\mathrm{NF}_{\kappa} \mathrm{B}$ p50 subunit was able to inhibit the nuclear import of transcription factors containing a NLS, such as $\mathrm{NF}_{\kappa} \mathrm{B}$, NFAT, AP-1 and STAT1 $(115,116)$. The peptide became better known as cSN50.1 and more recently has been found to target both SREBP/KPNB1- and KPNA/NLS-cargomediated import through two separate mechanisms. The inhibition of SREBP/KPNB1-mediated import is thought to be through the binding of the peptide's SSHR motif with KPNB1. The peptide is also able to bind all KPNA isoforms, except KPNA6, with the highest affinity for KPNA1 and in that way plays a role in the import inhibition of transcription factors containing a NLS (103). Kosugi et al. also used peptide inhibitor design to target the KPNA/B1 import pathway which yielded two high-affinity peptides namely; Bimax1 and Bimax2. They were found to specifically target the KPNA-mediated import pathway and had no effect of KPNB1-only mediated import although they showed no specificity for Karyopherin alpha isoforms in mammalian cells (105). Unfortunately, the chemotherapeutic ability of these import inhibitors remains unknown. A peptide, M9M, has also been designed to inhibit nuclear import but rather by targeting TNPO1 which recognises a PY-NLS (108). M9M was used to elucidate the role of TNP01 in mitosis but its role in cancer has not been explored (75). An antiparasitic antibiotic, Ivermectin, already on the market, has also been found to bind non-specifically to KPNB1/KPNA preventing binding to the cargo proteins (104). Although Ivermectin has been shown to have anticancer effects this seems to be through a mechanism of action unrelated to nuclear import inhibition (117).

Karyostatin 1A was the first small-molecule inhibitor of KPNB1-mediated nuclear import to be described. Its mechanism of action is thought to be through blocking the binding of
RanGTP to KPNB1 disrupting the KPNA/B1 import pathway (106). The potential of this compound including its anticancer effect has not been explored to date. Another small-molecule inhibitor of nuclear import, Importazole, developed by Soderholm et al., was found to interfere with the interaction between RanGTP and KPNB1 and specifically disrupts KPNB1mediated import without affecting Transportin or XP01mediated transport (107). Importazole successfully inhibited $\mathrm{NF} \kappa \mathrm{B}$ p65 nuclear localisation in myeloma cells and induced apoptosis without affecting normal plasma cells, showing promise as an anticancer compound (118). Although no studies have been published using nuclear import inhibitors in combination with currently available chemotherapeutic agents it may be an area of interest following the potential success of combination therapies with nuclear export inhibitors.

Although other members of the Karyopherin family, such as CSE1L, show promise as cancer therapeutic targets, no inhibitors are available for any of the other transport proteins as yet. The targeting of other nuclear transport proteins that have a more limited selection of cargoes may have value as anticancer strategies. For this to occur, their association with cancer requires further investigation.

\section{Conclusions and Perspectives}

Nuclear transport proteins play an essential role in cellular functioning. Hence, the increased reliance, of transformed and cancer cells, on the nuclear transport proteins for their increased metabolic demands warrants their usefulness as chemotherapeutic targets. More recently, the potential of nuclear transporters as cancer biomarkers has been investigated. For example, the secretion of certain Karyopherin proteins into the urine or serum of cancer patients shows diagnostic potential, while the differential expression of Karyopherin proteins in cancer tissue in comparison to normal tissue suggests that these proteins have potential as prognostic markers as well. Further research is required to elucidate the clinical potential of nuclear transport proteins as diagnostic, prognostic and therapeutic targets.

\section{Acknowledgements}

This work is supported through funding from the National Research Foundation (NRF), Cancer Association of South Africa (CANSA), the South African Medical Research Council (MRC), the Italian Association for Cancer Research and the University of Cape Town. Tamara Stelma is supported by the Harry Crossley Research Fellowship.

\section{References}

[1] Aggarwal, A. and Agrawal, D. K. (2014) Importins and exportins regulating allergic immune responses. Mediators Inflamm. 2014, 476357.

[2] Marfori, M., Mynott, A., Ellis, J. J., Mehdi, A. M., Saunders, N. F., et al. (2011) Molecular basis for specificity of nuclear import and prediction of nuclear localization. Biochim. Biophys. Acta 1813, 1562-1577. 
[3] van der Watt, P. J., Maske, C. P., Hendricks, D. T., Parker, I. M., Denny, L., et al. (2009) The Karyopherin proteins, Crm1 and Karyopherin beta1, are overexpressed in cervical cancer and are critical for cancer cell survival and proliferation. Int. J. Cancer 124, 1829-1840.

[4] Zhu, J., Wang, Y., Huang, H., Yang, Q., Cai, J., et al. (2015) Upregulation of KPNbeta1 in gastric cancer cell promotes tumor cell proliferation and predicts poor prognosis. Tumour Biol. Epub ahead of print, http://www.ncbi.nlm. nih.gov/pubmed/26242264.

[5] Kuusisto, H. V. and Jans, D. A. (2015) Hyper-dependence of breast cancer cell types on the nuclear transporter Importin beta1. Biochim. Biophys. Acta 1850, 1870-1878.

[6] Das, S. and Krainer, A. R. (2014) Emerging functions of SRSF1, splicing factor and oncoprotein, in RNA metabolism and cancer. Mol. Cancer Res. 12, 1195-1204.

[7] van der Watt, P. J., Ngarande, E., and Leaner, V. D. (2011) Overexpression of Kpnbeta1 and Kpnalpha2 importin proteins in cancer derives from deregulated E2F activity. PLoS One 6, e27723.

[8] Alshareeda, A. T., Negm, O. H., Green, A. R., Nolan, C. C., Tighe, P., et al. (2015) KPNA2 is a nuclear export protein that contributes to aberrant localisation of key proteins and poor prognosis of breast cancer. Br. J. Cancer 112, 1929-1937.

[9] Grupp, K., Habermann, M., Sirma, H., Simon, R., Steurer, S., et al. (2014) High nuclear karyopherin alpha 2 expression is a strong and independent predictor of biochemical recurrence in prostate cancer patients treated by radical prostatectomy. Mod. Pathol. 27, 96-106.

[10] Gousias, K., Niehusmann, P., Gielen, G. H., and Simon, M. (2014) Karyopherin a2 and chromosome region maintenance protein 1 expression in meningiomas: novel biomarkers for recurrence and malignant progression. J. Neurooncol. 118, 289-296.

[11] Altan, B., Yokobori, T., Mochiki, E., Ohno, T., Ogata, K., et al. (2013) Nuclear karyopherin-alpha2 expression in primary lesions and metastatic lymph nodes was associated with poor prognosis and progression in gastric cancer. Carcinogenesis 34, 2314-2321.

[12] Zheng, M., Tang, L., Huang, L., Ding, H., Liao, W. T., et al. (2010) Overexpression of karyopherin-2 in epithelial ovarian cancer and correlation with poor prognosis. Obstet. Gynecol. 116, 884-891.

[13] Jensen, J. B., Munksgaard, P. P., Sorensen, C. M., Fristrup, N., BirkenkampDemtroder, K., et al. (2011) High expression of karyopherin-alpha2 defines poor prognosis in non-muscle-invasive bladder cancer and in patients with invasive bladder cancer undergoing radical cystectomy. Eur. Urol. 59, 841-848.

[14] Shi, B., Su, B., Fang, D., Tang, Y., Xiong, G., et al. (2015) High expression of KPNA2 defines poor prognosis in patients with upper tract urothelial carcinoma treated with radical nephroureterectomy. BMC Cancer 15, 380.

[15] Jiang, P., Tang, Y., He, L., Tang, H., Liang, M., et al. (2014) Aberrant expression of nuclear KPNA2 is correlated with early recurrence and poor prognosis in patients with small hepatocellular carcinoma after hepatectomy. Med. Oncol. 31, 131.

[16] Winnepenninckx, V., Lazar, V., Michiels, S., Dessen, P., Stas, M., et al. (2006) Gene expression profiling of primary cutaneous melanoma and clinical outcome. J. Natl. Cancer Inst. 98, 472-482.

[17] Wang, C. I., Wang, C. L., Wang, C. W., Chen, C. D., Wu, C. C., et al. (2011) Importin subunit alpha-2 is identified as a potential biomarker for non-small cell lung cancer by integration of the cancer cell secretome and tissue transcriptome. Int. J. Cancer 128, 2364-2372.

[18] Sakai, M., Sohda, M., Miyazaki, T., Suzuki, S., Sano, A., et al. (2010) Significance of karyopherin-\{alpha\} 2 (KPNA2) expression in esophageal squamous cell carcinoma. Anticancer Res. 30, 851-856.

[19] Hügel, S., Depping, R., Dittmar, G., Rother, F., Cabot, R., et al. (2014) Identification of importin $\alpha 7$ specific transport cargoes using a proteomic screening approach. Mol. Cell Proteomics 13, 1286-1298.

[20] Laurila, E., Vuorinen, E., Savinainen, K., Rauhala, H., and Kallioniemi, A. (2014) KPNA7, a nuclear transport receptor, promotes malignant properties of pancreatic cancer cells in vitro. Exp. Cell Res. 322, 159-167.

[21] Ishizawa, J., Kojima, K., Hail, N., Jr., Tabe, Y., and Andreeff, M. (2015) Expression, function, and targeting of the nuclear exporter chromosome region maintenance 1 (CRM1) protein. Pharmacol. Ther. 153, 25-35.
[22] Zhou, F., Qiu, W., Yao, R., Xiang, J., Sun, X., et al. (2013) CRM1 is a novel independent prognostic factor for the poor prognosis of gastric carcinomas. Med. Oncol. 30, 726.

[23] Noske, A., Weichert, W., Niesporek, S., Roske, A., Buckendahl, A. C., et al. (2008) Expression of the nuclear export protein chromosomal region maintenance/exportin $1 / \mathrm{Xpo} 1$ is a prognostic factor in human ovarian cancer. Cancer 112, 1733-1743.

[24] Huang, W. Y., Yue, L., Qiu, W. S., Wang, L. W., Zhou, X. H., et al. (2009) Prognostic value of CRM1 in pancreas cancer. Clin. Invest. Med. 32, E315.

[25] Yao, Y., Dong, Y., Lin, F., Zhao, H., Shen, Z., et al. (2009) The expression of CRM1 is associated with prognosis in human osteosarcoma. Oncol. Rep. 21, 229-235.

[26] Kojima, K., Kornblau, S. M., Ruvolo, V., Dilip, A., Duvvuri, S., et al. (2013) Prognostic impact and targeting of CRM1 in acute myeloid leukemia. Blood 121, 4166-4174.

[27] van der Watt, P. J., Zemanay, W., Govender, D., Hendricks, D. T., Parker, M. I., et al. (2014) Elevated expression of the nuclear export protein, Crm1 (exportin 1), associates with human oesophageal squamous cell carcinoma. Oncol. Rep. 32, 730-738.

[28] Brinkmann, U., Brinkmann, E., Gallo, M., and Pastan, I. (1995) Cloning and characterization of a cellular apoptosis susceptibility gene, the human homologue to the yeast chromosome segregation gene CSE1. Proc. Natl. Acad. Sci. USA 92, 10427-10431.

[29] Tai, C. J., Liao, C. F., Su, T. C., Shen, K. H., Chang, C. C., et al. (2012) Presence of CSE1L protein in urine of patients with urinary bladder urothelial carcinomas. Int. J. Biol. Markers 27, e280-e284.

[30] Chang, C. C., Tai, C. J., Su, T. C., Shen, K. H., Lin, S. H., et al. (2012) The prognostic significance of nuclear CSE1L in urinary bladder urothelial carcinomas. Ann. Diagn. Pathol. 16, 362-368.

[31] Brinkmann, U., Brinkmann, E., Gallo, M., Scherf, U., and Pastan, I. (1996) Role of CAS, a human homologue to the yeast chromosome segregation gene CSE1, in toxin and tumor necrosis factor mediated apoptosis. Biochemistry 35, 6891-6899.

[32] Shiraki, K., Fujikawa, K., Sugimoto, K., Ito, T., Yamanaka, T., et al. (2006) Cellular apoptosis susceptibility protein and proliferation in human hepatocellular carcinoma. Int. J. Mol. Med. 18, 77-81.

[33] Stella Tsai, C. S., Chen, H. C., Tung, J. N., Tsou, S. S., Tsao, T. Y., et al. (2010) Serum cellular apoptosis susceptibility protein is a potential prognostic marker for metastatic colorectal cancer. Am. J. Pathol. 176, 1619-1628.

[34] Stawerski, P., Wagrowska-Danilewicz, M., Stasikowska, O., and Danilewicz, M. (2010) Immunoexpression of CAS protein is augmented in high grade serous ovarian tumors. Pol. J. Pathol. 61, 219-223.

[35] Wellmann, A., Krenacs, L., Fest, T., Scherf, U., Pastan, I., et al. (1997) Localization of the cell proliferation and apoptosis-associated CAS protein in lymphoid neoplasms. Am. J. Pathol. 150, 25-30.

[36] Melo, S. A., Moutinho, C., Ropero, S., Calin, G. A., Rossi, S., et al. (2010) A genetic defect in exportin-5 traps precursor microRNAs in the nucleus of cancer cells. Cancer Cell 18, 303-315.

[37] Gontan, C., Güttler, T., Engelen, E., Demmers, J., Fornerod, M., et al. (2009) Exportin 4 mediates a novel nuclear import pathway for Sox family transcription factors. J. Cell Biol. 185, 27-34.

[38] Kurisaki, A., Kurisaki, K., Kowanetz, M., Sugino, H., Yoneda, Y., et al. (2006) The mechanism of nuclear export of Smad3 involves Exportin 4 and Ran. Mol. Cell Biol. 26, 1318-1332.

[39] Liang, X. T., Pan, K., Chen, M. S., Li, J. J., Wang, H., et al. (2011) Decreased expression of XPO4 is associated with poor prognosis in hepatocellular carcinoma. J. Gastroenterol. Hepatol. 26, 544-549.

[40] Kimura, M. and Imamoto, N. (2014) Biological significance of the importinbeta family-dependent nucleocytoplasmic transport pathways. Traffic 15, 727-748.

[41] Pumroy, R. A. and Cingolani, G. (2015) Diversification of importin-alpha isoforms in cellular trafficking and disease states. Biochem. J. 466, 13-28.

[42] Poon, I. K. and Jans, D. A. (2005) Regulation of nuclear transport: central role in development and transformation? Traffic 6, 173-186. 
[43] Stewart, M. (2007) Molecular mechanism of the nuclear protein import cycle. Nat. Rev. Mol. Cell Biol. 8, 195-208.

[44] Kau, T. R., Way, J. C., and Silver, P. A. (2004) Nuclear transport and cancer: from mechanism to intervention. Nat. Rev. Cancer 4, 106-117.

[45] Forbes, D. J., Travesa, A., Nord, M. S., and Bernis, C. (2015) Nuclear transport factors: global regulation of mitosis. Curr. Opin. Cell Biol. 35, 78-90.

[46] Mosammaparast, N. and Pemberton, L. F. (2004) Karyopherins: from nuclear-transport mediators to nuclear-function regulators. Trends Cell Biol. $14,547-556$.

[47] Depping, R., Schindler, S. G., Jacobi, C., Kirschner, K. M., and Scholz, H. (2012) Nuclear transport of Wilms' tumour protein Wt1 involves importins alpha and beta. Cell Physiol. Biochem. 29, 223-232.

[48] Kuusisto, H. V., Wagstaff, K. M., Alvisi, G., Roth, D. M., and Jans, D. A. (2012) Global enhancement of nuclear localization-dependent nuclear transport in transformed cells. FASEB J. 26, 1181-1193.

[49] Smith, E. R., Cai, K. Q., Smedberg, J. L., Ribeiro, M. M., Rula, M. E., et al. (2010) Nuclear entry of activated MAPK is restricted in primary ovarian and mammary epithelial cells. PLoS One 5, e9295.

[50] Huang, L., Wang, H. Y., Li, J. D., Wang, J. H., Zhou, Y., et al. (2013) KPNA2 promotes cell proliferation and tumorigenicity in epithelial ovarian carcinoma through upregulation of $\mathrm{c}-\mathrm{Myc}$ and downregulation of FOXO3a. Cell Death Dis. 4, e745.

[51] Wang, C. I., Chien, K. Y., Wang, C. L., Liu, H. P., Cheng, C. C., et al. (2012) Quantitative proteomics reveals regulation of karyopherin subunit alpha-2 (KPNA2) and its potential novel cargo proteins in nonsmall cell lung cancer. Mol. Cell. Proteomics 11, 1105-1122.

[52] Nevins, J. R. (2001) The Rb/E2F pathway and cancer. Hum. Mol. Genet. 10, 699-703.

[53] Lin, J., Zhang, L., Huang, H., Huang, Y., Huang, L., et al. (2015) MiR-26b/ KPNA2 axis inhibits epithelial ovarian carcinoma proliferation and metastasis through downregulating OCT4. Oncotarget 15, 23793-23806.

[54] Quan, Y., Ji, Z. L., Wang, X., Tartakoff, A. M., and Tao, T. (2008) Evolutionary and transcriptional analysis of karyopherin beta superfamily proteins. Mol. Cell. Proteomics 7, 1254-1269.

[55] Kahle, J., Baake, M., Doenecke, D., and Albig, W. (2005) Subunits of the heterotrimeric transcription factor NF-Y are imported into the nucleus by distinct pathways involving importin beta and importin 13. Mol. Cell Biol. 25, 5339-5354.

[56] Muller, P. A. and Vousden, K. H. (2013) p53 mutations in cancer. Nat. Cell Biol. 15, 2-8.

[57] Winkler, J., Ori, A., Holzer, K., Sticht, C., Dauch, D., et al. (2014) Prosurvival function of the cellular apoptosis susceptibility/importin-alpha1 transport cycle is repressed by p53 in liver cancer. Hepatology 60, 884-895.

[58] Kim, I. S., Kim, D. H., Han, S. M., Chin, M. U., Nam, H. J., et al. (2000) Truncated form of importin alpha identified in breast cancer cell inhibits nuclear import of p53. J. Biol. Chem. 275, 23139-23145.

[59] Kutay, U., Bischoff, F. R., Kostka, S., Kraft, R., and Gorlich, D. (1997) Export of importin alpha from the nucleus is mediated by a specific nuclear transport factor. Cell 90, 1061-1071.

[60] Lorenzato, A., Martino, C., Dani, N., Oligschlager, Y., Ferrero, A. M., et al. (2012) The cellular apoptosis susceptibility CAS/CSE1L gene protects ovarian cancer cells from death by suppressing RASSF1C. FASEB J. 26, 24462456.

[61] Scherf, U., Kalab, P., Dasso, M., Pastan, I., and Brinkmann, U. (1998) The hCSE1/CAS protein is phosphorylated by HeLa extracts and MEK-1: MEK-1 phosphorylation may modulate the intracellular localization of CAS. Biochem. Biophys. Res. Commun. 250, 623-628.

[62] King, F. W. and Shtivelman, E. (2004) Inhibition of nuclear import by the proapoptotic protein CC3. Mol. Cell Biol. 24, 7091-7101.

[63] Yu, Y., Xu, F., Peng, H., Fang, X., Zhao, S., et al. (1999) NOEY2 (ARHI), an imprinted putative tumor suppressor gene in ovarian and breast carcinomas. Proc. Natl. Acad. Sci. USA 96, 214-219.

[64] Huang, S., Chang, I. S., Lin, W., Ye, W., Luo, R. Z., et al. (2010) ARHI (DIRAS3), an imprinted tumour suppressor gene, binds to importins and blocks nuclear import of cargo proteins. Biosci. Rep. 30, 159-168.
[65] Gorlich, D., Seewald, M. J., and Ribbeck, K. (2003) Characterization of Randriven cargo transport and the RanGTPase system by kinetic measurements and computer simulation. EMBO J. 22, 1088-1100.

[66] Smith, A. E., Slepchenko, B. M., Schaff, J. C., Loew, L. M., and Macara, I. G. (2002) Systems analysis of Ran transport. Science 295, 488-491.

[67] Rensen, W. M., Mangiacasale, R., Ciciarello, M., and Lavia, P. (2008) The GTPase Ran: regulation of cell life and potential roles in cell transformation. Front. Biosci. 13, 4097-4121.

[68] Wiese, C., Wilde, A., Moore, M. S., Adam, S. A., Merdes, A., et al. (2001) Role of importin-beta in coupling Ran to downstream targets in microtubule assembly. Science 291, 653-656.

[69] Sillje, H. H., Nagel, S., Korner, R., and Nigg, E. A. (2006) HURP is a Ranimportin beta-regulated protein that stabilizes kinetochore microtubules in the vicinity of chromosomes. Curr. Biol. 16, 731-742.

[70] Blower, M. D., Nachury, M., Heald, R., and Weis, K. (2005) A Rae1containing ribonucleoprotein complex is required for mitotic spindle assembly. Cell 121, 223-234.

[71] Albee, A. J., Tao, W., and Wiese, C. (2006) Phosphorylation of maskin by Aurora-A is regulated by RanGTP and importin beta. J. Biol. Chem. 281, 38293-38301.

[72] Dikovskaya, D., Li, Z., Newton, I. P., Davidson, I., Hutchins, J. R., et al. (2010) Microtubule assembly by the Apc protein is regulated by importin-betaRanGTP. J. Cell Sci. 123, 736-746.

[73] Song, L., Craney, A., and Rape, M. (2014) Microtubule-dependent regulation of mitotic protein degradation. Mol. Cell 53, 179-192.

[74] Lau, C. K., Delmar, V. A., Chan, R. C., Phung, Q., Bernis, C., et al. (2009) Transportin regulates major mitotic assembly events: from spindle to nuclear pore assembly. Mol. Biol. Cell 20, 4043-4058.

[75] Bernis, C., Swift-Taylor, B., Nord, M., Carmona, S., Chook, Y. M., et al. (2014) Transportin acts to regulate mitotic assembly events by target binding rather than Ran sequestration. Mol. Biol. Cell 25, 992-1009.

[76] Liao, C. F., Luo, S. F., Shen, T. Y., Lin, C. H., Chien, J. T., et al. (2008) CSE1L/ CAS, a microtubule-associated protein, inhibits taxol (paclitaxel)-induced apoptosis but enhances cancer cell apoptosis induced by various chemotherapeutic drugs. BMB Rep. 41, 210-216.

[77] Behrens, P., Brinkmann, U., and Wellmann, A. (2003) CSE1L/CAS: its role in proliferation and apoptosis. Apoptosis 8, 39-44.

[78] Quensel, C., Friedrich, B., Sommer, T., Hartmann, E., and Kohler, M. (2004) In vivo analysis of importin alpha proteins reveals cellular proliferation inhibition and substrate specificity. Mol. Cell Biol. 24, 1024610255.

[79] Gruss, O. J., Carazo-Salas, R. E., Schatz, C. A., Guarguaglini, G., Kast, J., et al. (2001) Ran induces spindle assembly by reversing the inhibitory effect of importin alpha on TPX2 activity. Cell 104, 83-93.

[80] Hutchins, J. R., Moore, W. J., Hood, F. E., Wilson, J. S., Andrews, P. D., et al. (2004) Phosphorylation regulates the dynamic interaction of RCC1 with chromosomes during mitosis. Curr. Biol. 14, 1099-1104.

[81] Li, H. Y. and Zheng, Y. (2004) Phosphorylation of RCC1 in mitosis is essential for producing a high RanGTP concentration on chromosomes and for spindle assembly in mammalian cells. Genes Dev. 18, 512-527.

[82] Wu, Z., Jiang, Q., Clarke, P. R., and Zhang, C. (2013) Phosphorylation of Crm 1 by CDK1-cyclin-B promotes Ran-dependent mitotic spindle assembly. J. Cell Sci. 126, 3417-3428.

[83] Dasso, M. (2006) Ran at kinetochores. Biochem. Soc. Trans. 34, 711-715.

[84] Wang, W., Budhu, A., Forgues, M., and Wang, X. W. (2005) Temporal and spatial control of nucleophosmin by the Ran-Crm1 complex in centrosome duplication. Nat. Cell Biol. 7, 823-830.

[85] Latvia, P. (2016) The GTPase RAN regulates multiple steps of the centrosome life cycle. Chromosome Res (In Press).

[86] Yang, J., Bardes, E. S., Moore, J. D., Brennan, J., Powers, M. A., et al. (1998) Control of cyclin B1 localization through regulated binding of the nuclear export factor CRM1. Genes Dev. 12, 2131-2143.

[87] Knauer, S. K., Bier, C., Habtemichael, N., and Stauber, R. H. (2006) The Survivin-Crm1 interaction is essential for chromosomal passenger complex localization and function. EMBO Rep. 7, 1259-1265. 
[88] Ma, S. and Zhao, X. (2014) KPNA2 is a promising biomarker candidate for esophageal squamous cell carcinoma and correlates with cell proliferation. Oncol. Rep. 32, 1631-1637.

[89] Gousias, K., Becker, A. J., Simon, M., and Niehusmann, P. (2012) Nuclear karyopherin a2: a novel biomarker for infiltrative astrocytomas. J. Neurooncol. 109, 545-553.

[90] Shen, A., Wang, Y., Zhao, Y., Zou, L., Sun, L., et al. (2009) Expression of CRM1 in human gliomas and its significance in p27 expression and clinical prognosis. Neurosurgery 65, 153-159; discussion 159-160.

[91] Zender, L., Xue, W., Zuber, J., Semighini, C. P., Krasnitz, A., et al. (2008) An oncogenomics-based in vivo RNAi screen identifies tumor suppressors in liver cancer. Cell 135, 852-864.

[92] Christiansen, A. and Dyrskjot, L. (2013) The functional role of the novel biomarker karyopherin alpha 2 (KPNA2) in cancer. Cancer Lett. 331, 18-23.

[93] Gousias, K., Niehusmann, P., Gielen, G., Simon, M., and Bostrom, J. (2014) KPNA2 predicts long term survival in patients with anaplastic oligoastrocytomas. J. Clin. Neurosci. 21, 1719-1724.

[94] Dahl, E., Kristiansen, G., Gottlob, K., Klaman, I., Ebner, E., et al. (2006) Molecular profiling of laser-microdissected matched tumor and normal breast tissue identifies karyopherin alpha2 as a potential novel prognostic marker in breast cancer. Clin. Cancer Res. 12, 3950-3960.

[95] Gluz, O., Wild, P., Meiler, R., Diallo-Danebrock, R., Ting, E., et al. (2008) Nuclear karyopherin alpha2 expression predicts poor survival in patients with advanced breast cancer irrespective of treatment intensity. Int. J. Cancer 123, 1433-1438.

[96] Mortezavi, A., Hermanns, T., Seifert, H. H., Baumgartner, M. K., Provenzano, M., et al. (2011) KPNA2 expression is an independent adverse predictor of biochemical recurrence after radical prostatectomy. Clin. Cancer Res. 17, 1111-1121.

[97] Hu, Z. Y., Yuan, S. X., Yang, Y., Zhou, W. P., and Jiang, H. (2014) Pleomorphic adenoma gene 1 mediates the role of karyopherin alpha 2 and has prognostic significance in hepatocellular carcinoma. J. Exp. Clin. Cancer Res. 33, 61.

[98] Yoshitake, K., Tanaka, S., Mogushi, K., Aihara, A., Murakata, A., et al. (2011) Importin-alpha1 as a novel prognostic target for hepatocellular carcinoma. Ann. Surg. Oncol. 18, 2093-2103.

[99] Angus, L., van der Watt, P. J., and Leaner, V. D. (2014) Inhibition of the nuclear transporter, Kpnbeta1, results in prolonged mitotic arrest and activation of the intrinsic apoptotic pathway in cervical cancer cells. Carcinogenesis 35, 1121-1131.

[100] Alnabulsi, A., Agouni, A., Mitra, S., Garcia-Murillas, I., Carpenter, B., et al. (2012) Cellular apoptosis susceptibility (chromosome segregation 1-like, CSE1L) gene is a key regulator of apoptosis, migration and invasion in colorectal cancer. J. Pathol. 228, 471-481.

[101] Zhu, J. H., Hong, D. F., Song, Y. M., Sun, L. F., Wang, Z. F., et al. (2013) Suppression of cellular apoptosis susceptibility (CSE1L) inhibits proliferation and induces apoptosis in colorectal cancer cells. Asian Pac. J. Cancer Prev. 14, 1017-1021.

[102] Martens-de Kemp, S. R., Nagel, R., Stigter-van Walsum, M., van der Meulen, I. H., van Beusechem, V. W., et al. (2013) Functional genetic screens identify genes essential for tumor cell survival in head and neck and lung cancer. Clin. Cancer Res. 19, 1994-2003.

[103] Zienkiewicz, J., Armitage, A., and Hawiger, J. (2013) Targeting nuclear import shuttles, importins/karyopherins alpha by a peptide mimicking the
NFkappaB1/p50 nuclear localization sequence. J. Am. Heart Assoc. 2, e000386.

[104] Wagstaff, K. M., Rawlinson, S. M., Hearps, A. C., and Jans, D. A. (2011) An AlphaScreen(R)-based assay for high-throughput screening for specific inhibitors of nuclear import. J. Biomol. Screen 16, 192-200.

[105] Kosugi, S., Hasebe, M., Entani, T., Takayama, S., Tomita, M., et al. (2008) Design of peptide inhibitors for the importin alpha/beta nuclear import pathway by activity-based profiling. Chem. Biol. 15, 940-949.

[106] Hintersteiner, M., Ambrus, G., Bednenko, J., Schmied, M., Knox, A. J., et al. (2010) Identification of a small molecule inhibitor of importin beta mediated nuclear import by confocal on-bead screening of tagged onebead one-compound libraries. ACS Chem. Biol. 5, 967-979.

[107] Soderholm, J. F., Bird, S. L., Kalab, P., Sampathkumar, Y., Hasegawa, K., et al. (2011) Importazole, a small molecule inhibitor of the transport receptor importin-beta. ACS Chem. Biol. 6, 700-708.

[108] Cansizoglu, A. E., Lee, B. J., Zhang, Z. C., Fontoura, B. M., and Chook, Y. M. (2007) Structure-based design of a pathway-specific nuclear import inhibitor. Nat. Struct. Mol. Biol. 14, 452-454.

[109] Kudo, N., Wolff, B., Sekimoto, T., Schreiner, E. P., Yoneda, Y., et al. (1998) Leptomycin $\mathrm{B}$ inhibition of signal-mediated nuclear export by direct binding to CRM1. Exp. Cell Res. 242, 540-547.

[110] Lapalombella, R., Sun, Q., Williams, K., Tangeman, L., Jha, S., et al. (2012) Selective inhibitors of nuclear export show that CRM1/XPO1 is a target in chronic lymphocytic leukemia. Blood 120, 4621-4634.

[111] Parikh, K., Cang, S., Sekhri, A., and Liu, D. (2014) Selective inhibitors of nuclear export (SINE)-a novel class of anti-cancer agents. J. Hematol. Oncol. 7, 78.

[112] Turner, J. G., Dawson, J., Cubitt, C. L., Baz, R., and Sullivan, D. M. (2014) Inhibition of CRM1-dependent nuclear export sensitizes malignant cells to cytotoxic and targeted agents. Semin. Cancer Biol. 27, 62-73.

[113] Turner, J. G., Dawson, J., Emmons, M. F., Cubitt, C. L., Kauffman, M., et al. (2013) CRM1 inhibition sensitizes drug resistant human myeloma cells to topoisomerase II and proteasome inhibitors both in vitro and ex vivo. J. Cancer 4, 614-625.

[114] Kazim, S., Malafa, M. P., Coppola, D., Husain, K., Zibadi, S., et al. (2015) Selective nuclear export inhibitor KPT-330 enhances the antitumor activity of gemcitabine in human pancreatic cancer. Mol. Cancer Ther. 14, 1570-1581.

[115] Lin, Y. Z., Yao, S. Y., Veach, R. A., Torgerson, T. R., and Hawiger, J. (1995) Inhibition of nuclear translocation of transcription factor NF-kappa B by a synthetic peptide containing a cell membrane-permeable motif and nuclear localization sequence. J. Biol. Chem. 270, 14255-14258.

[116] Torgerson, T. R., Colosia, A. D., Donahue, J. P., Lin, Y. Z., and Hawiger., J. (1998) Regulation of NF-kappa B, AP-1, NFAT, and STAT1 nuclear import in $\mathrm{T}$ lymphocytes by noninvasive delivery of peptide carrying the nuclear localization sequence of NF-kappa B p50. J. Immunol. 161, 6084-6092.

[117] Hashimoto, H., Messerli, S. M., Sudo, T., and Maruta, H. (2009) Ivermectin inactivates the kinase PAK1 and blocks the PAK1-dependent growth of human ovarian cancer and NF2 tumor cell lines. Drug Discov. Ther. 3, 243246.

[118] Yan, W., Li, R., He, J., Du, J., and Hou, J. (2015) Importin $\beta 1$ mediates nuclear factor $-\kappa \mathrm{B}$ signal transduction into the nuclei of myeloma cells and affects their proliferation and apoptosis. Cell Signal. 27, 851-859. 